\title{
Fault Detection for Switched Positive Systems under Successive Packet Dropouts with Application to the Leslie Matrix Model
}

\author{
Dong Wang, Zidong Wang, Guoyang Li and Wei Wang
}

\begin{abstract}
In this paper, the problem of fault detection filter design is dealt with for a class of switched positive systems with packet dropouts on the channel between the sensors and the filters. The phenomena of packet dropouts are governed by a Bernoulli process and a stochastic switched positive system is established based on the augmented states of the plants and filters. Two criteria are developed to evaluate the performance of the fault detection for the system under investigation. Sufficient conditions are established on the existence of the desired filters for the mean-square stability with an $L_{1}$ disturbance attenuation level and an index for the $L_{-}$fault sensitivity is also derived through constructing a switched Lyapunov function in term of linear programming. Two illustrative examples, one of which is concerned with the Leslie matrix model, are provided to show the effectiveness and applicability of the proposed results.
\end{abstract}

\section{Index Terms}

Switched positive system, Packet dropouts, Fault detection, Leslie matrix model.

\section{INTRODUCTION}

A switched system is a dynamical system that consists of a number of subsystems and a switching signal indicating a subsystem being activated during a certain interval. Switched positive systems are a special kind of switched systems whose states are nonnegative. Such systems are universal in practice such as networks employing transmission control protocol (TCP) [22] and rival mutation treatment dynamics [14]. Due to their significance in both theory and applications [11], [19]-[21], [32], [33], there has been an increasing interest in the control problems of switched positive systems, and some methods have been developed along the similar line of those for switched systems such as the average dwell time technology [19], the multiple Lyapunov function approach [21] and the switched Lyapunov function approach [20].

With the increasing demand for high safety and reliability standards in industrial process, the problem of fault detection (FD) has been a hot topic of research for three decades [6], [10], [15]. An extensive

This work is in part supported by the National Natural Science Foundation of China under Grants 61104114, 61201035, 61374070 and 61473055, and the Fundamental Research Funds for the Central Universities in China under Grants DUT14QY14 and DUT14QY31.

D. Wang, G. Li and W. Wang are with the Faculty of Electronic Information and Electrical Engineering, Dalian University of Technology, Dalian 116024, P. R. China. (e-mails: wangdongandy@gmail.com;li_guoyang@sina.com; wangwei@dlut.edu.cn).

Z. Wang is with the Department of Computer Science, Brunel University London, Uxbridge, Middlesex, UB8 3PH, U.K. He is also with the Faculty of Engineering, King Abdulaziz University, Jeddah 21589, Saudi Arabia. (Email: Zidong.Wang@brunel.ac.uk) 
and effective method is to design fault detection systems based on the plant mathematical model [3], [13], [17], [24], [30]. The basic idea of such a method is to use state observers or filters to construct a residual signal and then compare it with a threshold. When the residual evaluation function has a value larger than the threshold, an alarm is generated. In practice, the parameter uncertainties and unknown disturbances are often mixed with the faults, and this can lead to false alarms. As such, the FD systems would have to be sensitive to faults and simultaneously robust to disturbance inputs as well as parameter uncertainties. Accordingly, some relevant results have been reported [15], [18], [25], where the main idea is to transform the FD problem into an $H_{-} / H_{\infty}$ or $H_{\infty}$ filtering problem [1], [2], [24], [25]. This idea has recently been extended to switched positive systems. For example, an $L_{1}$-filtering design problem for a continuous-time switched positive delay system has been studied in [30] and the fault detection of positive switched systems with time-varying delay has been achieved by designing a mixed $L_{-} / L_{\infty}$ observer via the delta operator approach in [18].

On the other hand, in the transmission and processing of measurement data from the sensors to the filter/estimator/detector, the packet dropout (or missing measurements) is usually unavoidable due mainly to the limited bandwidth of the channels and temporal sensor failures. Clearly, the packet dropout would degrade the system performance and its impact on the fault detection problem has been an emerging challenging problem [16]. So far, a common approach to modelling the packet dropouts has been the use of a random variable satisfying the Bernoulli binary distribution taking values on either 1 or 0 , where 1 implies the perfect signal delivery and 0 represents the packet dropout. The fault detection problem with packet dropouts has received considerable research attention and many important results have been reported in recent years [7], [12], [13], [28]. In particular, such a problem has been dealt with in networked systems with distributed sensors [12]. To the best of the authors' knowledge, the FD problem for discrete-time switched positive systems with packet dropouts has not gained adequate attention yet, and this motivates the current investigation with hope to demonstrate the application potential in practical engineering.

In this paper, the FD problem for switched positive systems subject to random packet dropouts is investigated, where the Bernoulli distributions are employed to describe the phenomena of the packet dropouts. When the packet dropout occurs, the data received at the last time instant is used for updating the filter input. This way, the $L_{-}$and $L_{1}$ performance indices are established to evaluate the sensitivity of generated residuals to fault signals in faulty cases and the robustness against the disturbance. By virtue of linear programming technique, sufficient conditions are presented on the existence of fault detection filters. Finally, two examples are provided to show the effectiveness of the proposed results, where the practical example is concerned with the Leslie matrix model as one of representative switched positive systems.

This paper is organized as follows. A system description is given and the fault detection problem of switched positive systems with packet dropouts is formulated in Section II. Two types of performance indices (i.e., the $L_{-}$and $L_{1}$ indices) of the fault detection system are analyzed in Section III. Sufficient conditions on the existence of fault detection filters are presented in Section IV, followed by examples and conclusion in Section V and Section VI, respectively. 
Notations: The notation used in this paper is fairly standard. $A \succeq 0(\preceq, \succ, \prec)$ means that all entries of matrix $A$ are nonnegative (nonpositive, positive, negative ). $\operatorname{diag}\{\cdots\}$ indicates a block-diagonal matrix. The superscript $T$ and -1 stand for the transposition of vectors or matrices and the inverse matrix of nonsingular square matrix, respectively. $R\left(R^{+}\right), R_{n}\left(R_{n}^{+}\right)$and $R_{n \times n}\left(R_{n \times n}^{+}\right)$denote the set of the real (positive real) scalars, $n$ dimensional vectors and $n \times n$ dimensional matrices in the Euclidean space with the norm $\|x\|=\left(x^{T} x\right)^{1 / 2}$ and $E\{\cdot\}$ represents the mathematical expectation operator. $1_{n}$ is $\left[\begin{array}{lll}1 & \cdots & 1\end{array}\right]_{1 \times n}^{T} . l_{1}[0, \infty)$ is the space of summable infinite sequences with the norm $\|w\|_{1}=\sum_{k=0}^{n}\left|w_{k}\right|$.

\section{Problem Formulation}

Consider the discrete-time switched positive systems described by

$$
\begin{aligned}
x_{k+1} & =A_{\sigma_{k}} x_{k}+B_{\sigma_{k}} w_{k}+G_{\sigma_{k}} f_{k} \\
y_{k} & =C_{\sigma_{k}} x_{k}+D_{\sigma_{k}} f_{k}
\end{aligned}
$$

where $x_{k} \in R_{n}$ is the system state, $y_{k} \in R_{m}$ is the measured output, $w_{k}$ is the disturbance and $f_{k}$ is the fault belonging to the $L_{1}$ space. $\sigma_{k}:[0, \infty) \rightarrow \Delta=\{1,2 \ldots N\}$ is an arbitrary switching sequence with $N$ being the number of subsystems. For $\sigma_{k}=i$, the $i$ th subsystem is active with its parameters $A_{i}, B_{i}, G_{i}, D_{i}$ and $C_{i}$ being positive constant matrices or vectors with appropriate dimensions. The initial condition $x_{0}$ is assumed to be nonnegative.

Definition 1 ( [21] ) System (1) is said to be positive if, for the nonnegative initial conditions $x_{0}, w_{k}$ and $f_{k}$ and arbitrary switching signals $\sigma_{k}$, the corresponding state trajectory $x_{k} \geq 0$ and output $y_{k} \geq 0$ hold for all $k \geq 0$.

Lemma 1 ( [21] ) System (1) is positive if and only if $A_{i} \succeq 0, B_{i} \succeq 0, C_{i} \succeq 0, D_{i} \succeq 0$ and $G_{i} \succeq 0, i \in \Delta$.

An FD system consists of a residual generator and a residual evaluation stage including an evaluation function and a threshold. For the purpose of residual generation, the following fault detection filter is constructed as a residual generator

$$
\begin{aligned}
\hat{x}_{k+1} & =\hat{A}_{\sigma_{k}} \hat{x}_{k}+\hat{B}_{\sigma_{k}} \bar{y}_{k} \\
r_{k} & =\hat{C}_{\sigma_{k}} \hat{x}_{k}+\hat{D}_{\sigma_{k}} \bar{y}_{k}
\end{aligned}
$$

where $\hat{x}_{k}$ is the state vector of a fault detection filter, $r_{k}$ is the so-called residual signal, $\bar{y}_{k}$ is the system measurement output received by a filter, $\hat{A}_{i}, \hat{B}_{i}, \hat{C}_{i}$ and $\hat{D}_{i}$ are positive matrices to be determined.

In practical systems, it is often the case that the packet dropout is inevitable because of resource (i.e., bandwidth and energy) constraint, intermittent sensor failures, etc. One of the approaches to dealing with such phenomenon is to assume that the package dropout is governed by a Bernoulli distributed sequence. In the present paper, the measurement output $y_{k}$ is transmitted to the filter (2). Let's introduce the Bernoulli variable sequence $\alpha_{k}$ satisfying the probability distribution law as follows:

$$
\begin{aligned}
& \operatorname{Prob}\left\{\alpha_{k}=1\right\}=E\left\{\alpha_{k}\right\}=\bar{\alpha} \\
& \operatorname{Prob}\left\{\alpha_{k}=0\right\}=1-E\left\{\alpha_{k}\right\}=1-\bar{\alpha} .
\end{aligned}
$$


If $y_{k}$ is successfully received by the filter, then $\alpha_{k}=1$, otherwise $\alpha_{k}=0$ (i.e., the measurement output is lost or the packet dropout occurs). In case of the packet dropouts, a natural method is to employ the last available measurement and the received data is described by

$$
\bar{y}_{k}=\alpha_{k} y_{k}+\left(1-\alpha_{k}\right) \bar{y}_{k-1} \text {. }
$$

Based on (1), (2) and (3), the system states are augmented as $\tilde{x}_{k}^{T}=\left[\begin{array}{lll}x_{k}^{T} & \hat{x}_{k}^{T} & \bar{y}_{k}^{T}\end{array}\right]$ and the resulting system becomes

$$
\begin{aligned}
\tilde{x}_{k+1} & =\tilde{A}_{\sigma_{k}} \tilde{x}_{k}+\tilde{B}_{\sigma_{k}} w_{k}+\tilde{G}_{\sigma_{k}} f_{k}+\left(\alpha_{k}-\bar{\alpha}\right)\left(\bar{A}_{\sigma_{k}} \tilde{x}_{k}+\bar{G}_{\sigma_{k}} f_{k}\right) \\
r_{k} & =\tilde{C}_{\sigma_{k}} \tilde{x}_{k}+\tilde{D}_{\sigma_{k}} f_{k}+\left(\alpha_{k}-\bar{\alpha}\right)\left(\bar{C}_{\sigma_{k}} \tilde{x}_{k}+\bar{D}_{\sigma_{k}} f_{k}\right)
\end{aligned}
$$

where

$$
\begin{aligned}
& \tilde{A}_{\sigma_{k}}=\left[\begin{array}{ccc}
A_{\sigma_{k}} & 0 & 0 \\
\bar{\alpha} \hat{B}_{\sigma_{k}} C_{\sigma_{k}} & \hat{A}_{\sigma_{k}} & (1-\bar{\alpha}) \hat{B}_{\sigma_{k}} \\
\bar{\alpha} C_{\sigma_{k}} & 0 & (1-\bar{\alpha}) I
\end{array}\right] \\
& \bar{A}_{\sigma_{k}}=\left[\begin{array}{ccc}
0 & 0 & 0 \\
\hat{B}_{\sigma_{k}} C_{\sigma_{k}} & 0 & -\hat{B}_{\sigma_{k}} \\
C_{\sigma_{k}} & 0 & -I
\end{array}\right] \\
& \tilde{B}_{\sigma_{k}}=\left[\begin{array}{c}
B_{\sigma_{k}} \\
0 \\
0
\end{array}\right] \\
& \tilde{C}_{\sigma_{k}}=\left[\begin{array}{lll}
\bar{\alpha} \hat{D}_{\sigma_{k}} C_{\sigma_{k}} & \hat{C}_{\sigma_{k}}(1-\bar{\alpha}) \hat{D}_{\sigma_{k}}
\end{array}\right] \\
& \bar{C}_{\sigma_{k}}=\left[\begin{array}{lll}
\hat{D}_{\sigma_{k}} C_{\sigma_{k}} & 0 & -\hat{D}_{\sigma_{k}}
\end{array}\right] \\
& \tilde{D}_{\sigma_{k}}=\bar{\alpha} \hat{D}_{\sigma_{k}} D_{\sigma_{k}} \text {, } \\
& \bar{D}_{\sigma_{k}}=\hat{D}_{\sigma_{k}} D_{\sigma_{k}} \\
& \tilde{G}_{\sigma_{k}}=\left[\begin{array}{c}
G_{k} \\
\bar{\alpha} \hat{B}_{\sigma_{k}} D_{\sigma_{k}} \\
\bar{\alpha} D_{\sigma_{k}}
\end{array}\right] \\
& \bar{G}_{\sigma_{k}}=\left[\begin{array}{c}
0 \\
\hat{B}_{\sigma_{k}} D_{\sigma_{k}} \\
D_{\sigma_{k}}
\end{array}\right] \text {. }
\end{aligned}
$$

The following definitions are introduced for quantifying the fault detection performance that is essential for our derivation.

Definition 2 ( $L_{1}$ performance). Given a positive scalar $\beta$, system (4) with $f(k)=0$ is said to satisfy a prescribed $L_{1}$ performance $\beta$ under zero-initial condition if

$$
E\left\{\sum_{k=0}^{\infty}\left\|r_{k}\right\|_{1}\right\} \leq \beta \sum_{k=0}^{\infty}\left\|w_{k}\right\|_{1}
$$

holds. 
Definition 3 ( $L_{-}$performance). Given a positive scalar $\gamma$, system (4) with $w(k)=0$ is said to satisfy a prescribed $L_{-}$performance $\gamma$ under zero-initial condition if

$$
E\left\{\sum_{k=0}^{\infty}\left\|r_{k}\right\|_{1}\right\} \geq \gamma \sum_{k=0}^{\infty}\left\|f_{k}\right\|_{1}
$$

holds.

Remark 1. The $L_{1}$ performance $\beta$ characterizes the disturbance attenuation level of the filter for the addressed switched positive system, which is similar to the frequently used $H_{\infty}$ index in the non-positive systems. On the other hand, the $L_{-}$performance $\gamma$ represents a measure for the fault sensitivity of the switched positive system, which is parallel to the $H_{-}$performance index in the non-positive systems. As a mixed performance index, $H_{-} / H_{\infty}$ has been proposed to deal with the fault detection problem for non-positive systems in [25] and, similarly, a mixed performance $L_{-} / L_{1}$ has been established to such a problem in the continuous time switched positive systems [18]. The present paper aims at solving such a problem in the discrete time switched positive systems.

For the residual evaluation stage, an evaluation function and a threshold are required. Here, the residual evaluation function is selected as [18]

$$
J_{r}(T)=\sum_{k=k_{0}}^{T}\left\|r_{k}\right\|_{1}
$$

where $T$ is the evaluation time window. Furthermore, whether a fault occurs or not is determined by the following logical relationship

$$
\begin{aligned}
J_{r}(T) & >J_{t h} \Rightarrow \text { Alarm } \\
J_{r}(T) & \leq J_{t h} \Rightarrow \text { No Alarm }
\end{aligned}
$$

where

$$
J_{t h}=\sup _{w_{k} \in l_{1}\left[k_{0}, \infty\right], f_{k}=0} J_{r}(T) .
$$

Remark 2. The selection of the evaluation time window $T$ is crucial to detect the occurred fault. If $T$ is relatively large, the sensitivity to detect the fault will be reduced while the false alarm rate will rise when $T$ becomes small. Therefore, it is of great importance to determine $T$ in accordance with the practical experience.

The objective of this paper is to design an FD filter (2) to detect the fault occurred in system (1) with random packet dropouts such that the augmented system (4) satisfies the following conditions:

1). System (4) is asymptotically stable and the effects of disturbances on residuals are minimized in the fault-free case, namely, the minimum of $\beta$ is found to satisfy $L_{1}$ performance requirement (5).

2 ). The effects of fault signal on residuals are maximized in the faulty case, namely, the maximum of $\gamma$ is found to satisfy $L_{-}$performance (6).

\section{FAult Detection AnAlysis}

In this section, a $L_{1} / L_{-}$fault detection scheme for switched positive systems is presented based on the switched Lyapunov function approach such that system (4) satisfies performance specifications (5) and (6). 


\section{A. $L_{1}$ performance}

The following theorem presents a sufficient condition under which the disturbance attenuation performance of (4) is guaranteed.

Theorem 1. Given a scalar $\beta>0$, system (4) with $f(k)=0$ is asymptotically stable and satisfies the performance specification (5) if there exist positive vectors $\vartheta_{i}$ and $i, j \in \Delta$ such that

$$
\begin{array}{r}
\tilde{A}_{i}^{T} \vartheta_{j}-\vartheta_{i}+\tilde{C}_{i}^{T} \preceq 0 \\
\tilde{B}_{i}^{T} \vartheta_{j}-\beta \preceq 0 .
\end{array}
$$

Proof. When $f(k)=0$, system (4) is transformed into

$$
\begin{aligned}
\tilde{x}_{k+1} & =\tilde{A}_{\sigma_{k}} \tilde{x}_{k}+\tilde{B}_{\sigma_{k}} w_{k}+\left(\alpha_{k}-\bar{\alpha}\right) \bar{A}_{\sigma_{k}} \tilde{x}_{k} \\
r_{k} & =\tilde{C}_{\sigma_{k}} \tilde{x}_{k}+\left(\alpha_{k}-\bar{\alpha}\right) \bar{C}_{\sigma_{k}} \tilde{x}_{k} .
\end{aligned}
$$

Select the following Lyapunov function

$$
V(k)=\tilde{x}_{k}^{T} \vartheta_{\sigma_{k}}
$$

where $\vartheta_{\sigma_{k}}$ are positive vectors. Then, the mathematical expectation of Lyapunov function (10) at the next time instant is

$$
\begin{aligned}
& E\{V(k+1) \mid V(k)\} \\
= & E\left\{\tilde{x}_{k}^{T} \tilde{A}_{\sigma_{k}}^{T} \vartheta_{\sigma_{k+1}}+\omega_{k}^{T} \tilde{B}_{\sigma_{k}}^{T} \vartheta_{\sigma_{k+1}}+\left(\alpha_{k}-\bar{\alpha}\right) \tilde{x}_{k}^{T} \bar{A}_{\sigma_{k}}^{T} \vartheta_{\sigma_{k+1}}\right\} \\
= & \tilde{x}_{k}^{T} \tilde{A}_{\sigma_{k}}^{T} \vartheta_{\sigma_{k+1}}+w_{k}^{T} \tilde{B}_{\sigma_{k}}^{T} \vartheta_{\sigma_{k+1}} .
\end{aligned}
$$

Using switched Lyapunov function method and calculating the forward difference of (10) along the trajectories of system (9) lead to

$$
\begin{aligned}
E\{\Delta V(k)\} & =E\{V(k+1) \mid V(k)\}-V(k) \\
& =\tilde{x}_{k}^{T}\left(\tilde{A}_{\sigma_{k}}^{T} \vartheta_{\sigma_{k+1}}-\vartheta_{\sigma_{k}}\right)+w_{k}^{T} \tilde{B}_{\sigma_{k}}^{T} \vartheta_{\sigma_{k+1}}
\end{aligned}
$$

It is seen from (11) that $E\{\Delta V(k)\}<0$ when $\tilde{A}_{i}^{T} \vartheta_{j}-\vartheta_{i} \preceq 0$ and $w_{k}=0$ are satisfied, which implies that system (4) without disturbances is asymptotically stable if (8) holds.

The following performance index is defined to obtain the $L_{1}$ performance of system (9):

$$
J_{1}=E\left\{\sum_{k=0}^{\infty}\left(\left\|r_{k}\right\|_{1}-\beta\left\|w_{k}\right\|_{1}\right)\right\} \text {. }
$$


Based on the zero-initial conditions $\tilde{x}_{0}^{T}=0$, and $V(k) \geq 0$, we can get

$$
\begin{aligned}
J_{1}= & E\left\{\sum_{k=0}^{\infty}\left(\left\|r_{k}\right\|_{1}-\beta\left\|w_{k}\right\|_{1}\right)\right\} \\
= & E\left\{\sum_{k=0}^{\infty}\left(\left\|r_{k}\right\|_{1}-\beta\left\|w_{k}\right\|_{1}\right)\right\}+E\left\{\sum_{k=0}^{\infty} \Delta V(k)\right\} \\
& -\lim _{k \rightarrow \infty} E\{V(k+1) \mid V(k)\} \\
\leq & \sum_{k=0}^{\infty} E\left\{\left\|r_{k}\right\|_{1}-\beta\left\|w_{k}\right\|_{1}+\Delta(V(k))\right\} \\
= & \sum_{k=0}^{\infty}\left\{\tilde{x}_{k}^{T}\left(\tilde{A}_{\sigma_{k}}^{T} \vartheta_{\sigma_{k+1}}-\vartheta_{\sigma_{k}}+\tilde{C}_{\sigma_{k}}^{T}\right)+w_{k}^{T}\left(\tilde{B}_{\sigma_{k}}^{T} \vartheta_{\sigma_{k+1}}-\beta\right)\right\} \\
= & \sum_{k=0}^{\infty} \xi_{k}^{T} \Xi_{k, k+1}
\end{aligned}
$$

where

$$
\begin{aligned}
\xi_{k}^{T} & =\left[\begin{array}{cc}
\tilde{x}_{k}^{T} & w_{k}^{T}
\end{array}\right] \\
\Xi_{k, k+1} & =\left[\begin{array}{c}
\tilde{A}_{\sigma_{k}}^{T} \vartheta_{\sigma_{k+1}}-\vartheta_{\sigma_{k}}+\tilde{C}_{\sigma_{k}}^{T} \\
\tilde{B}_{\sigma_{k}}^{T} \vartheta_{\sigma_{k+1}}-\beta
\end{array}\right] .
\end{aligned}
$$

Obviously, $J_{1} \leq 0$ holds if $\Xi_{k, k+1} \preceq 0$. Letting $\sigma_{k}=i$ and $\sigma_{k+1}=j$ based on (12), we can have the inequality (8) which means that, if (8) holds, system (4) in the fault-free case is asymptotically stable and the performance index (5) is ensured. The proof is complete.

It is observed that, the smaller the value of $\beta$ is, the worse the disturbance attenuation performance of the system would be. If $\beta$ becomes larger, the fault could be regarded as the disturbance resulting in the missing alarm.

\section{B. $L_{-}$performance}

The following theorem deals with the fault sensitivity performance $\gamma$.

Theorem 2. Given a scalar $\gamma>0$, system (4) with $w(k)=0$ satisfies the performance specification (6) if there exist positive vectors $v_{i}$ and $i, j \in \Delta$ such that

$$
\begin{gathered}
\tilde{A}_{i}^{T} v_{j}-v_{i}-\tilde{C}_{i}^{T} \preceq 0 \\
\tilde{G}_{i}^{T} v_{j}+\gamma-\tilde{D}_{i}^{T} \preceq 0
\end{gathered}
$$

holds.

Proof. When $w(k)=0$, system (4) is rewritten as

$$
\begin{aligned}
\tilde{x}_{k+1} & =\tilde{A}_{\sigma_{k}} \tilde{x}_{k}+\tilde{G}_{\sigma_{k}} f_{k}+\left(\alpha_{k}-\bar{\alpha}\right)\left(\bar{A}_{\sigma_{k}} \tilde{x}_{k}+\bar{G}_{\sigma_{k}} f_{k}\right) \\
r_{k} & =\tilde{C}_{\sigma_{k}} \tilde{x}_{k}+\tilde{D}_{\sigma_{k}} f_{k}+\left(\alpha_{k}-\bar{\alpha}\right)\left(\bar{C}_{\sigma_{k}} \tilde{x}_{k}+\bar{D}_{\sigma_{k}} f_{k}\right) .
\end{aligned}
$$

Choosing the following Lyapunov candidate

$$
V(k)=\tilde{x}_{k}^{T} v_{\sigma_{k}}
$$


where $v_{\sigma_{k}}$ are positive vectors, we have

$$
\begin{aligned}
& E\{V(k+1) \mid V(k)\} \\
= & \tilde{x}_{k}^{T} \tilde{A}_{\sigma_{k}}^{T} v_{\sigma_{k+1}}+f_{k}^{T} \tilde{G}_{\sigma_{k}}^{T} v_{\sigma_{k+1}} .
\end{aligned}
$$

Using switched Lyapunov function method and calculating the forward difference of (15) along the trajectories of system (14), we have

$$
\begin{aligned}
E\{\Delta V(k)\} & =E\{V(k+1) \mid V(k)\}-V(k) \\
& =\tilde{x}_{k}^{T}\left(\tilde{A}_{\sigma_{k}}^{T} v_{\sigma_{k+1}}-v_{\sigma_{k}}\right)+f_{k}^{T} \tilde{G}_{\sigma_{k}}^{T} v_{\sigma_{k+1}} .
\end{aligned}
$$

In order to obtain the $L_{-}$performance of system (14), the following performance index is defined

$$
J_{-}=E\left\{\sum_{k=0}^{\infty}\left(\left\|r_{k}\right\|_{1}-\gamma\left\|f_{k}\right\|_{1}\right)\right\} \text {. }
$$

Based on the zero-initial conditions $\tilde{x}_{0}^{T}=0$ and $V(k) \geq 0$, we obtain

$$
\begin{aligned}
J_{-}= & E\left\{\sum_{k=0}^{\infty}\left(\left\|r_{k}\right\|_{1}-\gamma\left\|f_{k}\right\|_{1}\right)\right\} \\
= & E\left\{\sum_{k=0}^{\infty}\left(\left\|r_{k}\right\|_{1}-\gamma\left\|f_{k}\right\|_{1}\right)\right\}-E\left\{\sum_{k=0}^{\infty} \Delta V(k)\right\} \\
& +\lim _{k \rightarrow \infty} E\{V(k+1) \mid V(k)\} \\
\geq & \sum_{k=0}^{\infty} E\left\{\left\|r_{k}\right\|_{1}-\gamma\left\|f_{k}\right\|_{1}-\Delta V(k)\right\} \\
= & \sum_{k=0}^{\infty}\left\{\tilde{x}_{k}^{T}\left(-\tilde{A}_{\sigma_{k}}^{T} v_{\sigma_{k+1}}+v_{\sigma_{k}}+\tilde{C}_{\sigma_{k}}^{T}\right)+f_{k}^{T}\left(-\tilde{G}_{\sigma_{k}}^{T} v_{\sigma_{k+1}}-\gamma+\tilde{D}_{\sigma_{k}}^{T}\right)\right\} \\
= & -\sum_{k=0}^{\infty} \varsigma_{k}^{T} \Theta_{k, k+1}
\end{aligned}
$$

where

$$
\begin{aligned}
\varsigma_{k}^{T} & =\left[\begin{array}{cc}
\tilde{x}_{k}^{T} & f_{k}^{T}
\end{array}\right] \\
\Theta_{k, k+1} & =\left[\begin{array}{c}
\tilde{A}_{\sigma_{k}}^{T} v_{\sigma_{k+1}}-v_{\sigma_{k}}-\tilde{C}_{\sigma_{k}}^{T} \\
\tilde{G}_{\sigma_{k}}^{T} v_{\sigma_{k+1}}+\gamma-\tilde{D}_{\sigma_{k}}^{T}
\end{array}\right] .
\end{aligned}
$$

It is obvious that $J_{-} \geq 0$ when $\Theta \preceq 0$ is satisfied. Letting $\sigma_{k}=i$ and $\sigma_{k+1}=j$ based on (16), we can have the inequality (13). It can now be concluded that, if (13) holds, the performance index (6) is satisfied and the proof is complete.

It is seen from Theorem 2 that $\gamma$ is regarded as a measure of the fault sensitivity [18]. If it is relatively small, the disturbance could be treated as a fault that leads to the false alarm.

Remark 3. In [25], a mixed performance $H_{-} / H_{\infty}$ has been proposed for the fault detection problem for non-positive systems. Such an idea has been applied to handle the continuous-time switched positive systems by designing a mixed $L_{-} / L_{1}$ observer via delta operator approach in [18]. In the present paper, this idea has been employed to deal with the discrete-time switched positive systems. In addition, the 
phenomenon of packet dropouts, which might contribute to the increase of the false alarm rate, is taken into account. To be more specific, if an FD filter is designed according to Theorem 1 and Theorem 2 with proper parameters $\beta$ and $\gamma$, then system (4) is sensitive to fault signals in the faulty cases and robust against the disturbance in the absence of faults.

\section{Design of Fault Detection Filters}

Now we are in a position to present a solution to the fault detection filters design problem.

Theorem 3. For system (1) and filter (2), let two positive scalars $\gamma$ and $\beta$ be given. The fault detection problem with packet dropouts is solvable if there exist positive matrices $K_{i 1} \in R_{n \times n}^{+}, K_{i 2} \in R_{1 \times n}^{+}, K_{i 3} \in$ $R_{1 \times n}^{+}, K_{i 4} \in R^{+}, v_{i 1} \in R_{n \times 1}^{+}, V_{2}=\left[v_{21}, v_{22}, \cdots, v_{2 n}\right]^{T} \in R_{n \times 1}^{+}, v_{i 3} \in R_{m \times 1}^{+}, \vartheta_{i 1} \in R_{n \times 1}^{+}, \vartheta_{i 3} \in R_{m \times 1}^{+}(i, j \in \Delta)$ such that system (4) satisfies performance specifications (5) and (6) with the following constraints:

$$
\begin{gathered}
A_{i}^{T} \vartheta_{j 1}+\bar{\alpha} C_{i}^{T} K_{j 2} 1_{n}+\bar{\alpha} C_{i}^{T} \vartheta_{j 3}-\vartheta_{i 1}+\bar{\alpha} C_{i}^{T} K_{i 4}^{T} \preceq 0 \\
(1-\bar{\alpha}) K_{j 2} 1_{n}+(1-\bar{\alpha}) \vartheta_{j 3}-\vartheta_{i 3}+(1-\bar{\alpha}) K_{i 4}^{T} \preceq 0 \\
A_{i}^{T} v_{j 1}+\bar{\alpha} C_{i}^{T} K_{j 2} 1_{n}+\bar{\alpha} C_{i}^{T} v_{j 3}-v_{i 1}-\bar{\alpha} C_{i}^{T} K_{i 4}^{T} \preceq 0 \\
(1-\bar{\alpha}) K_{j 2} 1_{n}+(1-\bar{\alpha}) v_{j 3}-v_{i 3}-(1-\bar{\alpha}) K_{i 4}^{T} \preceq 0 \\
G_{i}^{T} v_{j 1}+\bar{\alpha} D_{i}^{T} K_{j 2} 1_{n}+\bar{\alpha} D_{i}^{T} v_{j 3}+\gamma-\bar{\alpha} D_{i}^{T} K_{i 4}^{T} \preceq 0 \\
K_{i 1} 1_{n}-V_{2}-K_{i 3}^{T} \preceq 0 \\
K_{i 1} 1_{n}-V_{2}+K_{i 3}^{T} \preceq 0 \\
B_{i}^{T} \vartheta_{j 1}-\beta \preceq 0
\end{gathered}
$$

Moreover, if there is a feasible solution, the filter parameter matrices can be constructed as follows

$$
\begin{gathered}
\hat{A}_{i}^{T}=K_{i 1} \bar{V}_{2}^{-1}, \hat{B}_{i}^{T}=K_{i 2} \bar{V}_{2}^{-1} \\
\hat{C}_{i}=K_{i 3}, \hat{D}_{i}=K_{i 4}
\end{gathered}
$$

where $\bar{V}_{2}$ is a diagonal matrix $\operatorname{diag}\left\{v_{21}, v_{22} \cdots v_{2 n}\right\}$.

Proof. By Theorems 1 and 2, if system (4) under arbitrary switching signals satisfies (5) and (6), then the FD problem of system (1) is solvable by designing filter (2). Hence, sufficient conditions on the existence of filters have to guarantee (8) and (13). Defining

$$
\begin{gathered}
K_{i 1}=\hat{A}_{i}^{T} \bar{V}_{2}, K_{i 2}=\hat{B}_{i}^{T} \bar{V}_{2} \\
K_{i 3}=\hat{C}_{i}, K_{i 4}=\hat{D}_{i} \\
v_{i}=\left[\begin{array}{c}
v_{i 1} \\
V_{2} \\
v_{i 3}
\end{array}\right], \vartheta_{i}=\left[\begin{array}{c}
\vartheta_{i 1} \\
V_{2} \\
\vartheta_{i 3}
\end{array}\right]
\end{gathered}
$$

and substituting (19) into (17) lead to (8) and (13), which implies that if there exist positive matrices $K_{i 1} \in R_{n \times n}^{+}, K_{i 2} \in R_{1 \times n}^{+}, K_{i 3} \in R_{1 \times n}^{+}, K_{i 4} \in R_{1 \times 1}^{+}, v_{i 1} \in R_{n \times 1}^{+}, v_{i 3} \in R_{m \times 1}^{+}, \vartheta_{i 1} \in R_{n \times 1}^{+}, \vartheta_{i 3} \in R_{m \times 1}^{+}(i, j \in \Delta)$ such that (17) are satisfied, then the performance specifications (5) and (6) are guaranteed for system (4). As such, the system (4) is not only sensitive to fault signal but also robust against the disturbance. At 
last, by simple transformation of (19), the filter parameters can be obtained and thus the fault detection problem is solved. The proof is now complete.

Remark 4. It should be noted that a mixed $H_{-} / H_{\infty}$ fault detection problem has been investigated in [25]. However, because of the nonnegative property of positive systems, a straightforward application of FD filter designs for non-positive dynamical systems to positive systems is not applicable [18]. Instead, an $L_{-}$index as a new sensitivity measure of the residual signal to faults is introduced for the design of positive fault detection filter. It is required that the designed $L_{-} / L_{1}$ filter not only ensures the robustness against disturbance input $w_{k}$ and the sensitivity to fault input $f_{k}$ but also guarantees the positivity of the augmented system (4).

Remark 5. Compared with [18], our results have the following distinguishing features: (1) the discretetime system is considered in our paper while the continuous-time case has been dealt with in [18]; (2) our focus is to consider the effect of packet dropouts on FD problem, which is closer to engineering applications.

\section{ILlustrative EXAMPLES}

In this section, we provide two illustrative examples to verify the effectiveness of Theorem 3 .

Example 1. Consider system (1) with $n=2, N=2$ and matrices as follows:

Subsystem 1:

$$
\begin{aligned}
& A_{1}=\left[\begin{array}{cc}
0.1 & 0.15 \\
0.8 & 0.4
\end{array}\right], B_{1}=\left[\begin{array}{l}
0.2 \\
0.1
\end{array}\right], G_{1}=\left[\begin{array}{l}
0.1 \\
0.4
\end{array}\right] \\
& C_{1}=\left[\begin{array}{ll}
0.7 & 0
\end{array}\right], D_{1}=0.3
\end{aligned}
$$

Subsystem 2:

$$
\begin{aligned}
& A_{2}=\left[\begin{array}{ll}
0.3 & 0.2 \\
0.9 & 0.5
\end{array}\right], B_{2}=\left[\begin{array}{l}
0.5 \\
0.3
\end{array}\right], G_{2}=\left[\begin{array}{c}
0.15 \\
0.2
\end{array}\right] \\
& C_{2}=\left[\begin{array}{ll}
0.4 & 0
\end{array}\right], D_{2}=0.6 .
\end{aligned}
$$

Given $\bar{\alpha}=0.7, \beta=8$ and $\gamma=0.1$, by Theorem 3, a feasible solution for (17) is found:

$$
\begin{aligned}
K_{11} & =\left[\begin{array}{ll}
4.9466 & 3.2491 \\
3.0209 & 1.2796
\end{array}\right], K_{12}=\left[\begin{array}{ll}
0.8072 & 0.8072
\end{array}\right] \\
K_{13} & =\left[\begin{array}{ll}
1.4942 & 1.3388
\end{array}\right], K_{14}=4.0881 \\
K_{21} & =\left[\begin{array}{ll}
6.2803 & 2.9470 \\
3.2660 & 1.2159
\end{array}\right], K_{22}=\left[\begin{array}{ll}
0.5775 & 0.5775
\end{array}\right] \\
K_{23} & =\left[\begin{array}{ll}
1.1841 & 1.2840
\end{array}\right], K_{24}=4.1546 \\
V_{2} & =\left[\begin{array}{ll}
12.2610 & 7.8367
\end{array}\right]^{T} .
\end{aligned}
$$

The following filter parameters can be obtained from (18) : 


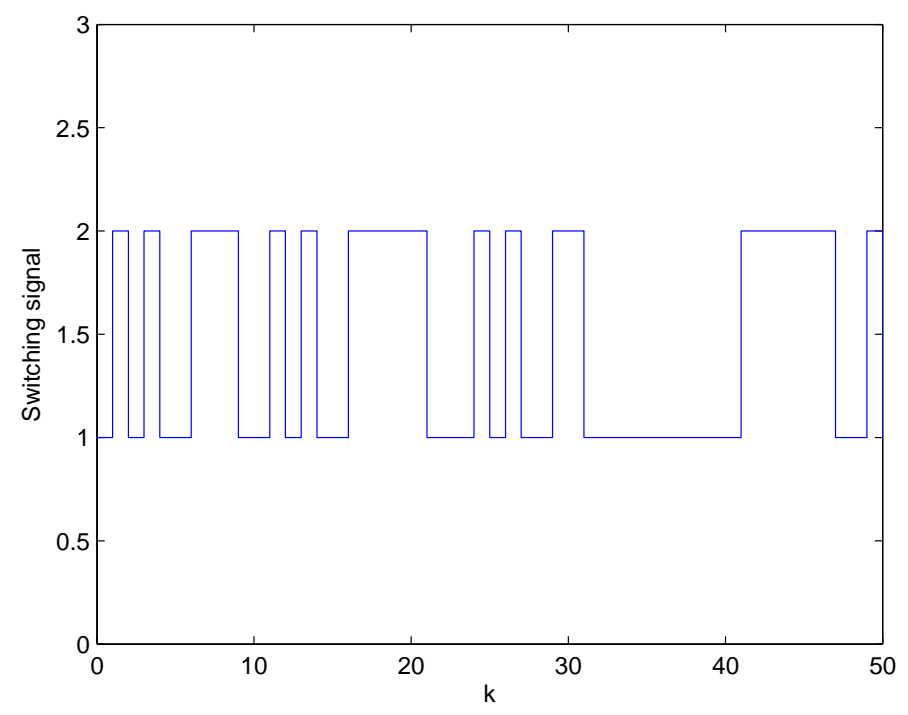

Fig. 1. Switching signal

Filter 1:

$$
\begin{aligned}
& \hat{A}_{1}=\left[\begin{array}{ll}
0.4034 & 0.2464 \\
0.4146 & 0.1633
\end{array}\right], \hat{B}_{1}=\left[\begin{array}{l}
0.0658 \\
0.1030
\end{array}\right] \\
& \hat{C}_{1}=\left[\begin{array}{ll}
1.4942 & 1.3388
\end{array}\right], \hat{D}_{1}=4.0881
\end{aligned}
$$

Filter 2:

$$
\begin{aligned}
& \hat{A}_{2}=\left[\begin{array}{ll}
0.5122 & 0.2664 \\
0.3761 & 0.1551
\end{array}\right], \hat{B}_{2}=\left[\begin{array}{l}
0.0471 \\
0.0737
\end{array}\right] \\
& \hat{C}_{2}=\left[\begin{array}{ll}
1.1841 & 1.2840
\end{array}\right], \hat{D}_{2}=4.1546 .
\end{aligned}
$$

The external disturbance is selected as the absolute value of a normal distribution with mean value 0 and variance 0.01 . The fault signal $f_{k}$ is set up as

$$
f_{k}=\left\{\begin{array}{c}
0.5, \quad 15 \leq k \leq 25 \\
0, \quad \text { others }
\end{array}\right.
$$

The initial state is chosen as $x_{0}=\left[\begin{array}{ll}2 & 4\end{array}\right]^{T}$. The switching signal, the states and the generated residual $r_{k}$ are shown in Fig. 1, Fig. 2 and Fig. 3, respectively. Fig. 4 displays the number of consecutive packet losses intuitively and Fig. 5 shows the evolution of residual evaluation function $J_{r}(T)$. The threshold can be determined as $J_{r}(T)=177.7655$ for $\mathrm{k}=50$ and the simulation results show that $J_{r}(19)=178.3691>$ 177.7655, which means that the fault $f_{k}$ can be detected after four sample time of its occurrence.

The simulation is provided to show the effectiveness of the proposed design methods. Though affected by the loss of data, the fault detection is achieved successfully. Next, a practical example is presented to the possible application.

Example 2. Consider a certain pest's structured population dynamics described by the Leslie matrix model named after British ecologist P. H. Leslie who popularized age structured models for animal 


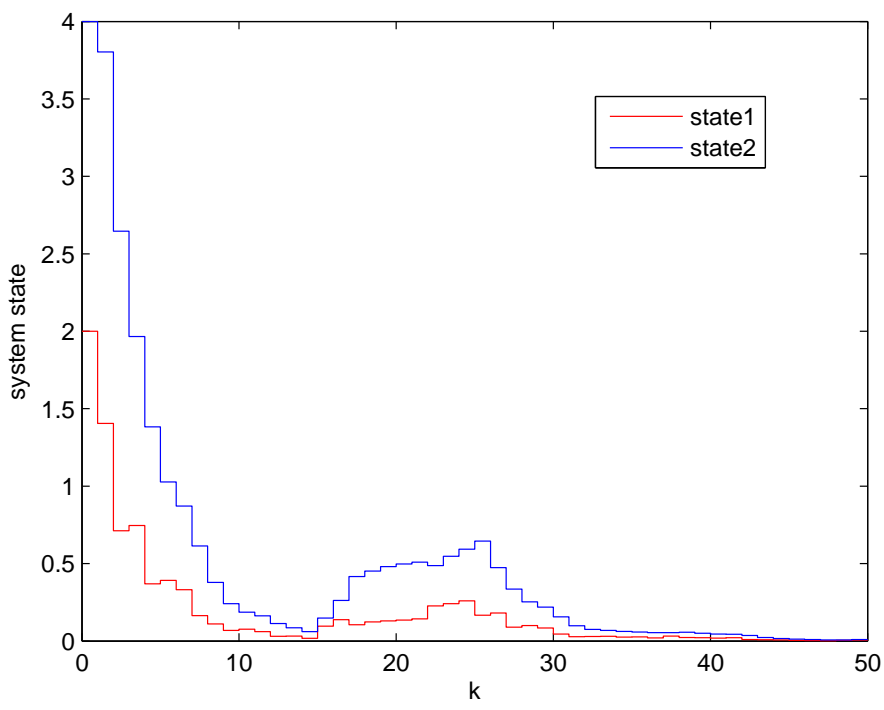

Fig. 2. States of the system

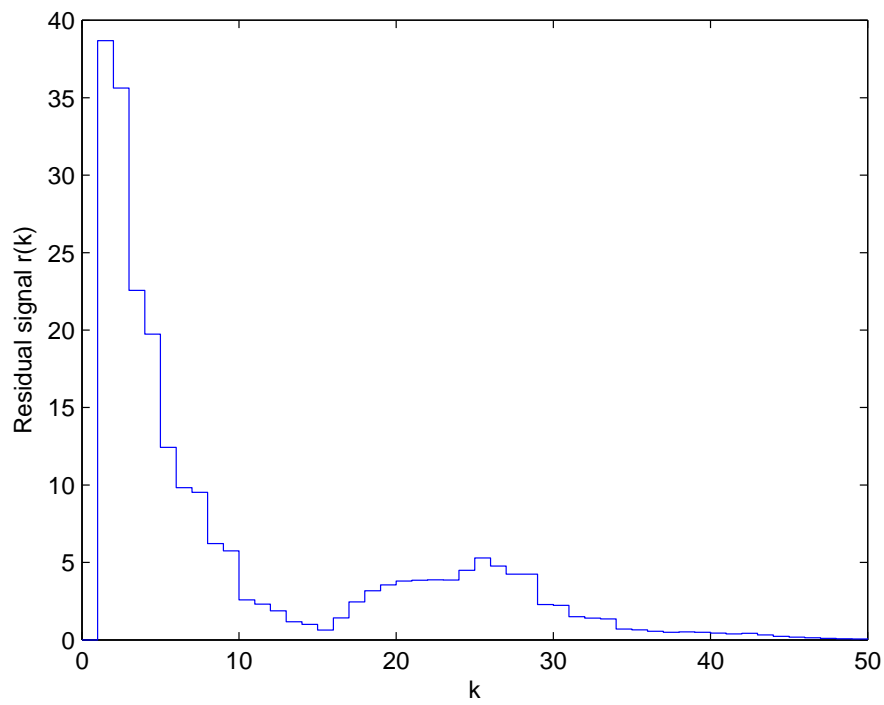

Fig. 3. Residual signal $r_{k}$

populations in the mid-twentieth century. The Leslie matrix model is the following [23]:

$$
\begin{aligned}
x_{k+1} & =\left[\begin{array}{ccc}
f_{1} & f_{2} & f_{3} \\
p_{1,2} & 0 & 0 \\
0 & p_{2,3} & 0
\end{array}\right] x_{k}+\left[\begin{array}{cc}
b_{1} & b_{2} \\
0 & 0 \\
0 & 0
\end{array}\right] u_{k} \\
y_{k} & =\left[\begin{array}{ccc}
0 & c & c
\end{array}\right] x_{k} \\
x_{k} & =\left[\begin{array}{lll}
x_{1}(k) & x_{2}(k) & x_{3}(k)
\end{array}\right]^{T}
\end{aligned}
$$

where $x_{1}(k)$ is the number of juvenile pests at time $\mathrm{k}, x_{2}(k)$ is the number of immature pests at time $\mathrm{k}$ and $x_{3}(k)$ is the number of adult pests at time k. $f_{i}$ denotes a birth rate for parents in the $i$ th age class 


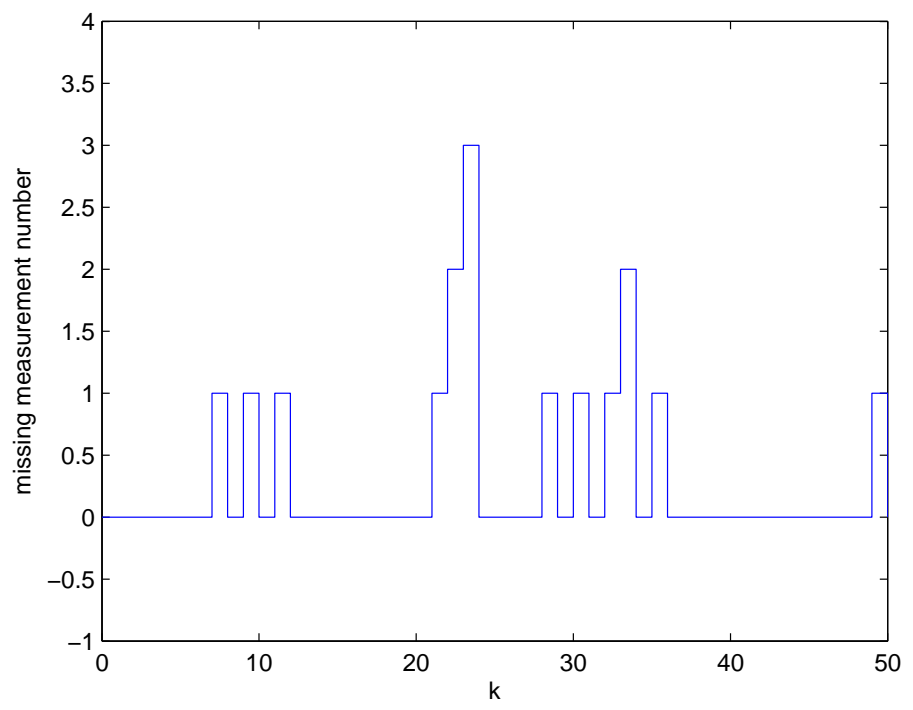

Fig. 4. The number of consecutive package dropout

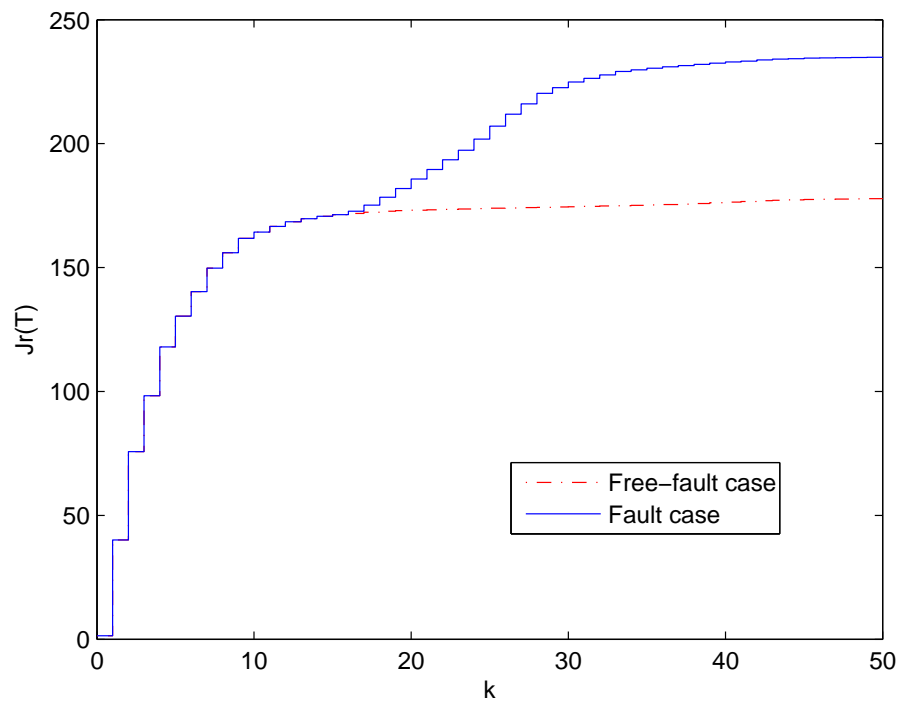

Fig. 5. Evolution of residual evaluation function $J_{r}(T)$

and $p_{i, j}, i, j \in\{1,2,3\}$ is a survival rate for those in the $i$ th age class passing into the $(i+1)$ th time instant. The structure of the input matrix means that only birth rates can be affected by external inputs. The output $y_{k}$ is the sum of number of immature and adult pests which can be observed. The parameters of the Leslie matrix model are

$$
\begin{gathered}
f_{1}=0.3011 \pm 0.0220, f_{2}=0.5915 \pm 0.0282 \\
f_{3}=0.5235 \pm 0.0022, p_{1,2}=0.8868 \pm 0.0128 \\
p_{2,3}=0.7894 \pm 0.0194, b_{1}=0.9000 \pm 0.0200 \\
b_{2}=0.5000 \pm 0.0050, c=1 \pm 0.01 .
\end{gathered}
$$


In practice, the number of pests of all ages can be reduced by spraying pesticide. Suppose that the state feedback controller is designed as $u_{k}=K x_{k}$ with

$$
K=\left[\begin{array}{rrr}
-0.1680 & -0.1192 & -0.0667 \\
-0.2438 & -0.1445 & -0.1563
\end{array}\right],
$$

where $K$ is related to the amount of pesticide.

Due to the existence of uncertainties in the system, it is able to model as a switched positive system with two subsystems,

$$
\begin{aligned}
& A_{1}=(\underline{\mathrm{A}}+\underline{\mathrm{B}} K)=\left[\begin{array}{ccc}
0.0106 & 0.3869 & 0.3896 \\
0.8740 & 0 & 0 \\
0 & 0.7700 & 0
\end{array}\right] \\
& A_{2}=(\bar{A}+\bar{B} K)=\left[\begin{array}{ccc}
0.0454 & 0.4371 & 0.3854 \\
0.8996 & 0 & 0 \\
0 & 0.8088 & 0
\end{array}\right] \\
& B_{w 1}=\left[\begin{array}{l}
0.60 \\
0.30 \\
0.83
\end{array}\right], B_{w 2}=\left[\begin{array}{l}
0.78 \\
0.34 \\
0.67
\end{array}\right] \\
& C_{1}=\left[\begin{array}{lll}
0 & 0.99 & 0.99
\end{array}\right] \\
& C_{2}=\left[\begin{array}{lll}
0 & 1.01 & 1.01
\end{array}\right] \\
& G_{1}=\left[\begin{array}{c}
0.105 \\
0.85 \\
0.87
\end{array}\right], G_{2}=\left[\begin{array}{l}
0.12 \\
0.45 \\
0.88
\end{array}\right] \\
& D_{1}=1.2, D_{2}=1.4 \text {. }
\end{aligned}
$$

Given $\bar{\alpha}=0.7, \beta=10$ and $\gamma=0.1$, by Theorem 3, a feasible solution for (17) is obtained:

$$
\begin{aligned}
& K_{11}=\left[\begin{array}{ccc}
8.7005 & 4.0282 & 6.2246 \\
14.6368 & 4.9573 & 4.9573 \\
7.7872 & 5.0176 & 5.0176
\end{array}\right], K_{12}=\left[\begin{array}{lll}
0.0539 & 0.0539 & 0.0539
\end{array}\right] \\
& K_{13}=\left[\begin{array}{lll}
3.1117 & 2.9465 & 2.9556
\end{array}\right], K_{14}=0.9006 \\
& K_{21}=\left[\begin{array}{ccc}
6.2345 & 9.0464 & 3.9701 \\
10.0352 & 6.8591 & 6.8591 \\
2.7153 & 7.1269 & 7.1269
\end{array}\right], K_{22}=\left[\begin{array}{lll}
0.0462 & 0.0462 & 0.0462
\end{array}\right] \\
& K_{23}=\left[\begin{array}{lll}
3.0261 & 3.1761 & 1.2003
\end{array}\right], K_{24}=0.6642 \\
& V_{2}=\left[\begin{array}{lll}
27.5049 & 32.5325 & 25.8349
\end{array}\right]^{T} \text {. }
\end{aligned}
$$

Thus, the filter parameters are obtained from (18): 
Filter 1:

$$
\begin{aligned}
& \hat{A}_{1}=\left[\begin{array}{lll}
0.3163 & 0.5322 & 0.2831 \\
0.1238 & 0.1524 & 0.1542 \\
0.2409 & 0.1919 & 0.1942
\end{array}\right], \hat{B}_{1}=\left[\begin{array}{l}
0.0020 \\
0.0017 \\
0.0021
\end{array}\right] \\
& \hat{C}_{1}=\left[\begin{array}{lll}
3.1117 & 2.9465 & 2.9556
\end{array}\right], \hat{D}_{1}=0.9006
\end{aligned}
$$

Filter 2:

$$
\begin{aligned}
& \hat{A}_{2}=\left[\begin{array}{lll}
0.2267 & 0.3648 & 0.0987 \\
0.2781 & 0.2108 & 0.2191 \\
0.1537 & 0.2655 & 0.2759
\end{array}\right], \hat{B}_{2}=\left[\begin{array}{l}
0.0017 \\
0.0014 \\
0.0018
\end{array}\right] \\
& \hat{C}_{2}=\left[\begin{array}{lll}
3.0261 & 3.1761 & 3.2003
\end{array}\right], \hat{D}_{2}=0.6642 .
\end{aligned}
$$

In order to draw the curves, some selections are made as follows. Since it is possible that there are pests coming into the testing area frequently, the external disturbance is selected as absolute value of normal distribution whose mean value is 0 and variance is 0.25 . The fault $f_{k}$ can be the controller failure or the mutant of state such as the failure of pesticides caused by rain after just spraying and the influx of a large number of pests from external region. Then, the fault signal $f_{k}$ is set up as

$$
f_{k}=\left\{\begin{array}{c}
e \times 5 \times(k-10), \quad 10 \leq k \\
0, \text { others }
\end{array}\right.
$$

where $e \in\{0,1\}$ is a flag number, $e=1$ indicates that the fault exists and $e$ is set to 0 if the fault is removed. The initial state is chosen as $x_{0}=\left[\begin{array}{lll}20 & 50 & 30\end{array}\right]^{T}$. Fig. 6 shows the random switching signal and Fig. 7 displays the number of consecutive package dropout. The number of immature pests $x_{2}(k)$ and adult pests $x_{3}(k)$ are shown in Fig. 8. Fig. 9 shows the evolution of residual evaluation function $J_{r}(T)$. The threshold can be determined as $J_{r}(T)=3069.0$ for $\mathrm{k}=60$ and the simulation results shows that $J_{r}(14)=3168.9>3069.0$, which means that the fault $f_{k}$ can be detected after four sample time of its occurrence.

In Example 2, we study a pest's population dynamics model that is of practical significance in agriculture and forestry. Since the Leslie matrix model is stabilized by designing a state feedback controller, it is unstable when the controller failure occurs. Once a fault is detected, some actions shall be taken in time. Otherwise, the number of pests will have a rapid growth resulting in economic losses. As shown in Fig. 8, after the fault happening at $k=10$, it is detected at $k=14$. Subsequently, measurements are taken to remove the fault and $e$ changes from 1 to 0 . Finally, pests are controlled effectively, which proves it is feasible to design the fault detection filter for Leslie matrix systems with packet dropouts in accordance with Theorem 3 .

\section{CONCLUSION}

The fault detection problem of switched positive systems with packet dropouts has been studied in the paper. The packet dropout is assumed to abide by the Bernoulli distributed (binary switching) white 


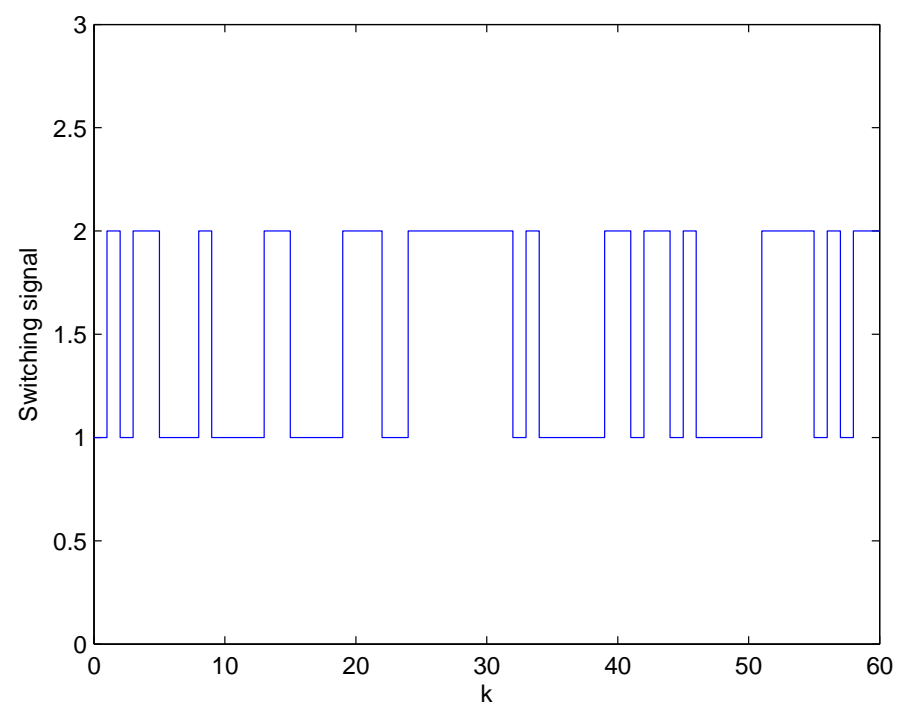

Fig. 6. Switching signal

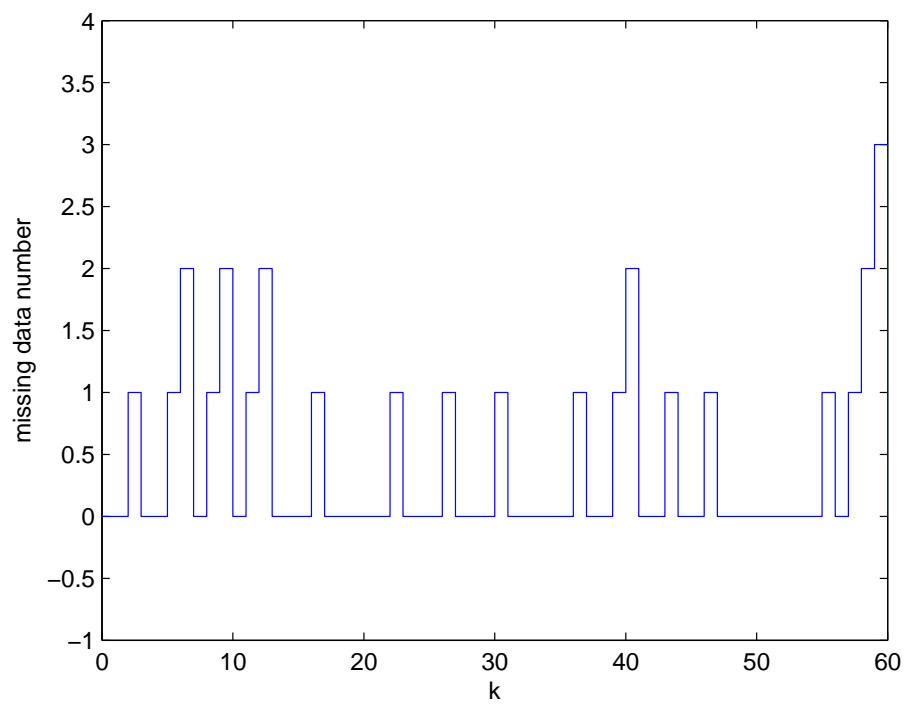

Fig. 7. The number of consecutive package dropout

sequence. By the algebraic operation, the stochastic information has been transferred into the parameters of an augmented stochastic dynamic system. Two criteria for the augmented systems have been utilized to address FD problem of the original system based on FD filter. Sufficient conditions for the existence of such filter have been obtained by constructing a switched Lyapunov function and the construction of filter has been provided. At last, the fault detection method has been applicable to the Leslie matrix model, which is very useful for agriculture and forestry. One of the future research topics is to extend the main results to more complicated systems [4], [5], [8], [9], [26], [27], [29], [31] such as stochastic systems, nonlinear systems and networked control systems. 


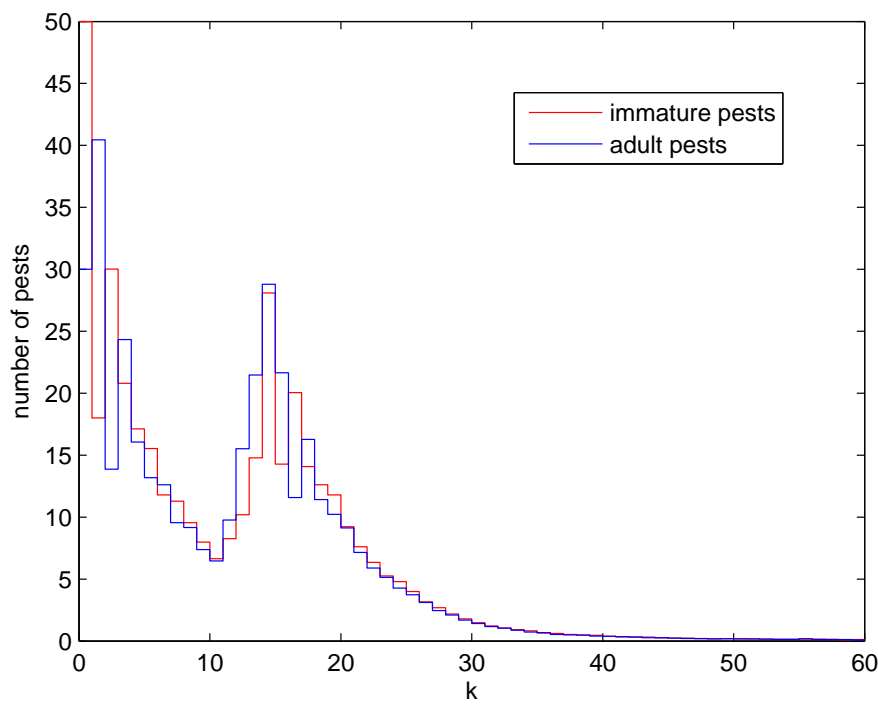

Fig. 8. Immature pests $x_{2}(k)$ and adult pests $x_{3}(k)$

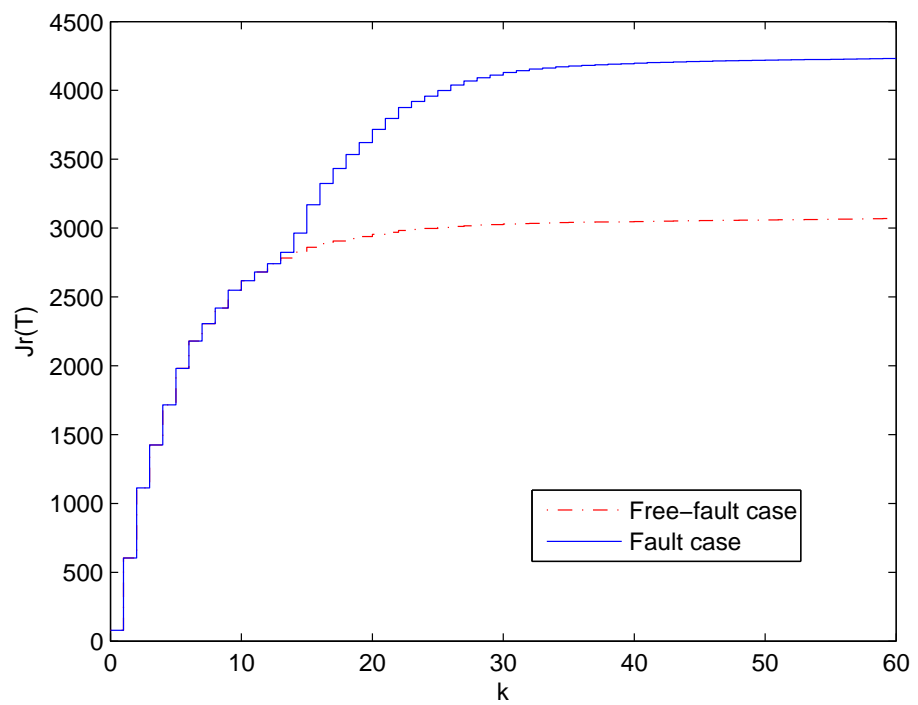

Fig. 9. Evolution of residual evaluation function $J_{r}(T)$

\section{REFERENCES}

[1] S. Aouaouda, M. Chadli, P. Shi and H. Karimi. Discrete-time $H_{-} / H_{\infty}$ sensor fault detection observer design for nonlinear systems with parameter uncertainty, International Journal of Robust Nonlinear Control, 25, 339-361, 2015.

[2] M. Basin, P. Shi and D. Calderon-Alvarez. Central suboptimal $H_{\infty}$ filter design for linear time-varying systems with state and measurement delays, International Journal of Systems Science, 41(4), 411-421, 2010.

[3] D. Belkhiat, N. Messai and N. Manamanni. A fault detection and isolation scheme for discrete time switched linear systems, 2011 9th IEEE International Conference on Control and Automation, Santiago, Dec. 9-11 2011, 596-601.

[4] D. Ding, Z. Wang, B. Shen and H. Dong, Envelope-constrained $H_{\infty}$ filtering with fading measurements and randomly occurring nonlinearities: the finite horizon case, Automatica, 55: 37-45, 2015.

[5] D. Ding, Z. Wang, J. Lam and B. Shen, Finite-Horizon $H_{\infty}$ control for discrete time-varying systems with randomly occurring nonlinearities and fading measurements, IEEE Transactions on Automatic Control, 60(9), 2488-2493, 2015. 
[6] S. Ding, Y. Yang, Y. Zhang and L. Li. Data-driven realizations of kernel and image representations and their application to fault detection and control system design, Automatica, 50(10), 2615-2623, 2014.

[7] H. Dong, Z. Wang, J. Lam and H. Gao. Fuzzy-model-based robust fault detection with stochastic mixed time delays and successive packet dropouts, IEEE Transactions on Systems, Man, and Cybernetics, Part B: Cybernetics, 42(2), 365-376, 2012.

[8] H. Dong, Z. Wang, S. X. Ding and H. Gao, Event-based H-infinity filter design for a class of nonlinear time-varying systems with fading channels and multiplicative noises, IEEE Transactions on Signal Processing, 63(13) 3387-3395, 2015.

[9] H. Dong, Z. Wang, S. X. Ding and H. Gao, Finite-horizon reliable control with randomly occurring uncertainties and nonlinearities subject to output quantization, Automatica, 52: 355-362, 2015.

[10] R. Ferrari, T. Parisini and M. Polycarpou. Distributed fault detection and isolation of large-scale discrete-time nonlinear systems: an adaptive approximation approach, IEEE Transactions on Automatic Control, 57(2), 275-290, 2012.

[11] E. Fornasini and M. Valcher. Asymptotic stability and stabilizability of special classes of discrete-time positive switched systems. Linear Algebra and its Applications, 438(4), 1814-1831, 2013.

[12] H. Gao, T. Chen and L. Wang. Robust fault detection with missing measurements, International Journal of Control, 81(5), 804-819, 2008 .

[13] X. He, Z. Wang and D. Zhou. Robust fault detection for networked systems with communication delay and data missing, Automatica, 45(11), 2634-2639, 2009.

[14] E. Hernandez-Vargas, P. Colaneri, R. Middleton and F. Blanchini. Discrete-time control for switched positive systems with application to mitigating viral escape, International Journal of Robust and Nonlinear Control, 21(10), 1093-1111, 2011.

[15] R. Isermann. Model-based fault-detection and diagnosis: status and applications, Annual Reviews in Control, 29(1), 71-85, 2005.

[16] S. Jiang and H. Fang. Robust fault detection for networked control systems with nonlinear disturbances and imperfect measurements, International Journal of Systems Science, 44(11), 2027-2038, 2013.

[17] H. Karimi. Robust synchronization and fault detection of uncertain master-slave systems with mixed time-varying delays and nonlinear perturbations, International Journal of Control, Automation and Systems, 9(4), 671-680, 2011.

[18] S. Li, Z. Xiang and H. Karimi. Mixed $l_{-} / l_{1}$ fault detection observer design for positive switched systems with time-varying delay via delta operator approach. International Journal of Control, Automation and Systems, 12(4), 709-721, 2014.

[19] J. Lian and J. Liu. New results on stability of switched positive systems: an average dwell-time approach, IET Control Theory and Applications, 7(12), 1651-1658, 2013.

[20] X. Liu. Stability analysis of switched positive systems: a switched linear copositive Lyapunov function method, IEEE Transactions on Circuits and Systems II: Express Briefs, 56(5), 414-418, 2009.

[21] O. Mason and R. Shorten. On linear copositive Lyapunov functions and the stability of switched positive linear systems, IEEE Transactions on Automatic Control, 52(7), 1346-1349, 2007.

[22] R. Shorten, F. Wirth and D. Leith. A positive systems model of TCP-like congestion control: asymptotic results, IEEE/ACM Transactions on Networking, 14(3), 616-629, 2006.

[23] Z. Shu, J. Lam and H. Gao. Positive observers and dynamic output-feedback controllers for interval positive linear systems, IEEE Transactions on Circuits and Systems I: Regular Papers, 55(10), 3209-3222, 2008.

[24] D. Wang, P. Shi and W. Wang. Robust fault detection for continuous-time switched delay systems: a linear matrix inequality approach, IET Control Theory and Applications, 4(1), 100-108, 2010.

[25] J. Wang, G. Yang and J. Liu. An LMI approach to $H_{-}$index and mixed $H_{-} / H_{\infty}$ fault detection observer design, Automatica, 43(9), 1656-1665, 2007.

[26] X. Wang, E. E. Yaz and J. Long, Robust and resilient state-dependent control of continuous-time nonlinear systems with general performance criteria, Systems Science and Control Engineering: An Open Access Journal, Vol. 2, No. 1, 2014, pp. 34-40.

[27] M. Lefebvre and F. Zitouni, Analytical solutions to LQG homing problems in one dimention, Systems Science and Control Engineering: An Open Access Journal, Vol. 2, No. 1, 2014, pp. 41-47.

[28] Z. Wang, B. Shen, H. Shu and G. Wei. Quantized $H_{\infty}$ control for nonlinear stochastic time-delay systems with missing measurements, IEEE Transactions on Automatic Control, 57(6), 1431-1444, 2012.

[29] G. Wei, S. Liu, Y. Song and Y. Liu, Probability-guaranteed set-membership filtering for systems with incomplete measurements, Automatica, Vol. 60, pp. 12-16, 2015.

[30] M. Xiang and Z. Xiang. Robust fault detection for switched positive linear systems with time-varying delays, ISA transactions, 54, 10-16, 2014.

[31] S. Zhang, Z. Wang, D. Ding, H. Dong, F. E. Alsaadi and T. Hayat, Non-fragile $H_{\infty}$ fuzzy filtering with randomly occurring gain variations and channel fadings, IEEE Transactions on Fuzzy Systems, 2015, in press, DOI: 10.1109/TFUZZ.2015.2446509 
[32] X. Zhao, L. Zhang, P. Shi and M. Liu. Stability of switched positive linear systems with average dwell time switching, Automatica, 48(6), 1132-1137, 2012.

[33] Y. Zheng and G. Feng. Two slow stabilizing switching laws for discrete time positive switched systems, International Journal of Robust and Nonlinear Control, 24, 2909-2927, 2014. 